\title{
OPEN Predictors of intensive care unit admission in patients with hematologic malignancy
}

\author{
Abi Vijenthira ${ }^{1}$, Nicholas Chiu ${ }^{2}$, Daniel Jacobson ${ }^{3}$, Zoey Freedman $^{4}$, Matthew C. Cheung ${ }^{5,6,7}$, \\ Shannon Goddard ${ }^{7}$, Robert Fowler ${ }^{8,9,10}$ \& Rena Buckstein ${ }^{6,7,11 \bowtie}$
}

Limited data exist on predictors of intensive care unit (ICU) admission in patients with hematologic malignancy. The objective of this study was to identify predictors of ICU admission in hospitalized patients with hematologic malignancies. A retrospective cohort study was conducted on 820 consecutive admissions of patients with a malignant hematology diagnosis at our institution between March 2009 and December 2015. Backward stepwise selection procedure was conducted for multivariable logistic regression analyses. 820 patients were included, of whom 179 (22\%) were admitted to the ICU. Types of hematologic cancers included $71 \%(\mathrm{~N}=578)$ lymphoid cancer, $18 \%$ ( $\mathrm{N}=151)$ myeloid cancer, and $10 \%(\mathrm{~N}=80)$ plasma cell neoplasms. $14 \%(\mathrm{~N}=111)$ of patients had acute leukemia. Six predictors of admission to ICU were found in multivariable analysis, including disease-related (acute leukemia, curative intent chemotherapy), laboratory-related (platelet count $<50 \times 109 / \mathrm{L}$, albumin below normal, $\mathrm{LDH}$ above normal at time of admission), and physicianrelated factors (having advanced directives discussion) $(p<0.0001)$. A significant proportion of patients with hematologic malignancies admitted to hospital are admitted to ICU. Utilizing the identified predictors of ICU admission may help guide timely informed goals of care discussions with patients before clinical deterioration occurs.

The survival of patients with hematologic malignancies has improved rapidly over the past several decades, owing to advances in diagnosis, therapy, and supportive care. However, the prognosis of patients admitted to the intensive care unit (ICU) with hematologic malignancies remains guarded, with contemporary data showing up to $46 \%-90 \%$ mortality in the in-hospital setting ${ }^{1-7}$, which is significantly higher than the mortality of contemporaneous general medical patients admitted to the $\mathrm{ICU}^{7}$. Predictors of ICU mortality have been highlighted in several papers, and include need for vasopressors, mechanical ventilation, and severity of illness ${ }^{1-6}$.

However, limited data exist on predictors of ICU admission in patients with hematologic malignancy. Previous studies have been limited by both substantial heterogeneity in patient populations - combining a majority of patients with solid tumors with lesser numbers of patients with hematologic malignancies ${ }^{8}$-or substantial specificity, restricting to patients with a particular hematologic diagnosis ${ }^{9}$.

The primary objective of this study was to identify predictors of ICU admission in hospitalized patients with hematologic malignancies to enable more informed goals of care conversations with these patients on admission. The secondary objectives were to describe the clinical outcomes and predictors of outcomes among patients with hematologic malignancies who were admitted to hospital.

\footnotetext{
${ }^{1}$ Division of Medical Oncology and Hematology, Princess Margaret Cancer Centre, Toronto, Canada. ${ }^{2}$ Boston University School of Medicine, Harvard T.H. Chan School of Public Health, Boston, USA. ${ }^{3}$ Western University and Ivey Business School, London, Canada. ${ }^{4}$ Postgraduate Genetics and Genome Sciences Education, Case Western Reserve University, Cleveland, USA. ${ }^{5}$ Evaluative Clinical Sciences, Odette Cancer Research Program, Sunnybrook Research Institute, Toronto, Canada. ${ }^{6}$ Department of Medicine, University of Toronto, Toronto, Canada. ${ }^{7}$ Division of Medical Oncology and Hematology, Odette Cancer Centre, Sunnybrook Health Sciences Centre, Toronto, Canada. ${ }^{8}$ Evaluative Clinical Sciences, Trauma, Emergency and Critical Care Research Program, Sunnybrook Research Institute, Toronto, Canada. ${ }^{9}$ Sunnybrook Health Sciences Centre, Toronto, Canada. ${ }^{10}$ Division of Critical Care Medicine, Department of Medicine and Interdepartmental, University of Toronto, Toronto, Canada. ${ }^{11}$ Odette Cancer Research Program, Sunnybrook Research Institute, Toronto, Canada. ${ }^{\boxplus}$ email: rena.buckstein@sunnybrook.ca
} 


\section{Methods}

A retrospective cohort study was conducted at a tertiary care centre in Toronto, Canada. Based on prior literature review, admission rate to ICU for patients with hematologic malignancies was estimated at $10 \%$. Thus, to attain an event rate of at least 100 to allow exploration of up to 10-20 predictors of the outcome of ICU admission, we planned to examine 1000 consecutive patient admissions with a malignant hematology diagnosis at our institution, using hospital-level health records from March 19, 2009 to December 29, 2015. An additional 27 consecutive patients admitted to the ICU from March 19-July 29, 2009 were added to improve statistical power. At our institution, once a malignant hematology diagnosis is established, subsequent admissions to hospital occur under the malignant hematology service, with the exception of patients admitted for scheduled surgery. Such patients would typically be transferred to the malignant oncology service after the immediate post-operative period. Patients were included if they had any diagnosis of a hematologic malignancy, and were excluded if the sole a priori reason for admission was transfer to palliative care. For patients admitted more than once during this time period, only the first admission was included. As the rate of ICU admission was higher than anticipated, the final sample size was reduced. Chart review was conducted by two research students (DJ and NC) and audited by ZF and RB.

Variables were collected from review of electronic medical records and included: age, sex, body mass index, comorbidities, primary reason for admission, hematologic malignancy diagnosis, time from diagnosis to admission, all prior chemo- and/or immunotherapy treatments, documented intent of therapy: incurable (e.g. indolent lymphomas, myelodysplastic syndrome [MDS]), versus curative (e.g. acute myeloid leukemia [AML], acute lymphocytic leukemia [ALL], diffuse large B-cell lymphoma [DLBCL]), timing from last chemotherapy received and granulocyte-colony stimulating factor (G-CSF) use. We recorded laboratory values at time of admission including hemoglobin (HGB), platelet count (PLT), white blood cell count (WBC), absolute neutrophil count (ANC), absolute lymphocyte count (ALC), creatinine (Cr) and calculated creatinine clearance, liver enzymes (AST, ALT, ALP), total bilirubin, albumin, lactate dehydrogenase (LDH). We recorded whether there was documentation of an advanced directives discussion at any time during hospitalization, or directives to not perform cardiopulmonary resuscitation (CPR). Individual diagnoses and total score of the modified Charlson Comorbidity Index ${ }^{10}$ were recorded. The study was approved by the Sunnybrook research ethics board (\#320-2017), and performed in accordance with the Tri-Council policies for ethical conduct in research. As this was a retrospective study, the need for informed consent was waived by the Sunnybrook research ethics board.

\section{Analysis}

Demographic and clinical characteristics of all patients were described, including for those with and without ICU admission, using counts, proportions, medians, interquartile ranges (IQR) for categorical and continuous variables as appropriate. To compare factors between groups, Wilcoxon rank-sum nonparametric test or Fisher's exact test were applied, as appropriate. To determine clinically important and statistically significant predictive factors of ICU admission and death in hospital or within 1 month of discharge, univariate logistic regression analysis was conducted. Because patients could be discharged or transferred to in-patient or home palliative care units, we categorized vital status as "death" for any death during hospitalization or until 30-days after hospital discharge. Natural log transformation was applied to certain covariates to normalize their distribution.

We used a backward stepwise selection procedure for multivariable logistic regression analyses and included any covariates with a $p$ value of $<0.10$ on univariate analysis. We considered several models with different covariates because of collinearity between these factors (e.g. PLT count $<50$ or $<100 \times 10^{9} / \mathrm{L}$, total comorbidity score and individual comorbidities). The model with the highest $\mathrm{R}^{2}$ was selected as the final model. Kaplan-Meier survival curves were graphed and compared by the log-rank test. Bonferroni corrected $p$ values $<0.002(0.05 / 32$ variables) were considered statistically significant for comparing the characteristics of patients admitted or not admitted to the ICU. $p$ value of $<0.05$ was considered statistically significant in the logistic regression analyses and log-rank test. All analyses were conducted using Statistical Analysis Software (SAS version 9.4 for Windows, Cary, NC) and R-package (version 3.5.0).

\section{Results}

Data were collected on 820 patients, of whom 179 (22\%) were admitted to the ICU. The median age was 66 years (IQR 56-76), and $44 \%$ were female $(\mathrm{n}=358)$. The median time from diagnosis to hospital admission was 6.7 months (IQR 2.3-36.9), and the median time from last chemotherapy use was 1 month (IQR 0-3).

The types of hematologic malignancies included: (a) lymphoid cancers, 578 patients ( $71 \%): 52$ patients $(6 \%)$ with lymphoid very aggressive (ALL, lymphoblastic lymphoma, Burkitt lymphoma), 327 patients (40\%) with lymphoid aggressive (e.g. DLBCL, peripheral T-cell lymphoma, anaplastic large cell lymphoma, primary central nervous system lymphoma etc.), and 199 patients (25\%) with lymphoid indolent (follicular, marginal zone, small lymphocytic, etc.); (b) myeloid cancers, 151 patients (18\%): 87 patients (11\%) with myeloid very aggressive (AML, blastic plasmacytoid dendritic cell neoplasm), 60 patients (7\%) with myeloid aggressive (MDS, chronic myelomonocytic leukemia, etc.), and 4 patients (0.5\%) with myeloid indolent (myeloproliferative neoplasms); (c) plasma cell neoplasms, 80 patients (10\%). Of the total sample of 820 patients, one hundred and eleven (14\%) patients had acute leukemia and $45(41 \%)$ were admitted to ICU. In contrast, only $21 \%(\mathrm{~N}=78)$ and $24 \%(\mathrm{~N}=19)$ of patients with very aggressive/aggressive lymphoma or plasma cell dyscrasias, respectively, were admitted to the ICU.

The most common causes for hospital admission were infections and febrile neutropenia $(\mathrm{N}=289,35 \%)$, followed by elective admission for chemotherapy $(\mathrm{N}=139,17 \%)$. Chemotherapy was administered to 262 patients (32\%) during their admission. Further details on types of hematologic cancers and reasons for admission are depicted in Supplementary Table S1. 
Seventy-three patients (41\%) admitted to the ICU received mechanical ventilation, 17 (9\%) received noninvasive ventilation, $8(4 \%)$ received dialysis and $85(47 \%)$ received vasoactive medications for blood pressure support. Eighty-four (47\%) and 95 (53\%) patients were admitted to high intensity and intermediate care units respectively.

Timing and length of stay. The median length of stay in hospital was 7 days for all patients (IQR 4-15). The median time from hospitalization to ICU admission was 3 days (IQR 1-10, range 0-49) and the median ICU length of stay was 2 days (IQR 1-5, range $0-23$ ). ICU admitted patients had longer total lengths of hospital stay than ward-alone patients (18 days (IQR 9-29) versus 5 days (IQR 3-10), $p<0.0001$ ).

Goals of care conversations. Among the 179 patients admitted to the ICU, 94 patients (53\%) had a documented conversation about goals of care prior to ICU admission and of these, 76 patients (80\%) chose full resuscitation in the event of cardiorespiratory failure. Among the 641 patients not admitted to the ICU, 208 patients (33\%) had a goals of care conversation and of these, 110 patients $(53 \%)$ chose full resuscitation.

Characteristics of patients admitted to ICU. Patients admitted to the ICU had shorter times from cancer diagnosis, lower hemoglobin, platelet count, creatinine clearance and albumin, and higher LDH upon hospital admission compared to those who remained on the ward. They were more likely to be red blood cell transfusion dependent before ICU admission and during their hospital stay and to have received non-curative intent chemotherapy. A high proportion of ICU admitted patients (115 patients, 64\%) had acute leukemia (myeloid predominated) or high-grade lymphomas. While not statistically significant after Bonferroni correction, ICU admitted patients had a higher proportion of elevated comorbidity scores ( $\mathrm{mCCI} \geq 2: 43 \% \mathrm{vs.} 30 \%, p=0.003$ ). Infection as a cause of admission was not associated with ICU admission (Table 1).

The covariates associated with ICU admission by univariate analysis are depicted in Supplementary Table S2. By multivariable analysis, the covariates most associated with ICU admission included chemotherapy intent (non-curative vs. curative), PLT $<50 \times 10^{9} / \mathrm{L}$, acute leukemia, albumin below normal limits $(<37 \mathrm{~g} / \mathrm{L}), \mathrm{LDH}$ above normal limits ( $>250 \mathrm{U} / \mathrm{L}$ ), and advanced directive discussion in hospital pre-ICU admission (yes versus no) (Table 2). Age was not significant in univariate analysis and thus was not tested in the multivariable model.

Overall survival. At a median follow-up of 8.1 months (IQR 1.5-25), a total of 328 patients (40\%) died in the study, including 179 (22\%) who died during hospitalization or within 1 month of discharge (Fig. 1). Of ICU admitted patients $(n=179), 43 \%$ had died within 1 month, including $37(21 \%)$ who died in the ICU, $35(19.6 \%)$ who died on the ward and $6(3 \%)$ who died within 1 month of discharge. Median time to death after ICU admission was 4.5 days overall (IQR 1-14) but only 1 day for those who died in the ICU (IQR 0-2, range 0-8). Of nonICU patients $(n=641), 16 \%$ had died within 1 month, including $45(7 \%)$ who died on the ward and $56(9 \%)$ who died within 1 month of discharge. Median time to death in non-ICU patients who died on the ward or within 1 month of discharge was 21 days (IQR 9-31) and 11 days (IQR 6-25) for death on the ward only. The patients admitted to the ICU had inferior overall survival with an actuarial survival of 3.7 months compared with not reached $p<0.0001$ (Fig. 1F).

By univariate analysis, predictors of death in hospital or within 1 month of discharge for ICU admitted patients included admission to a high intensity (versus step-down) ICU (OR 2.4, $p=0.004$ ), having had an advanced directives discussion (OR 1.9, $p=0.03$ ); higher than normal values of WBC (OR 1.2, $p=0.04)$, ALP (OR 1.9, $p=0.02$ ), LDH (OR 3.2, $p=0.01$ ), receiving mechanical ventilation (OR $4.8, p<0.0001$ ) or vasopressors (OR 2.1, $p=0.02$ ), and having a history of a solid tumor (OR 2.4, $p=0.03$ ). Age, BMI, mCCI and creatinine clearance were not predictive.

By multivariable analysis, $\mathrm{LDH}$ exceeding normal (OR 3.6, $p=0.02)$ and receiving mechanical ventilation at any time during hospitalization (OR $5.4, p=0.001$ ) were the only measures independently predictive of death in ICU admitted patients.

When considering all patients admitted to hospital, the covariates predictive of death in hospital (in ICU or ward) or within 1 month of discharge by multivariable analysis were: age (OR 1.03 per year of age, $p=0.005$ ), platelet count $<50 \times 10^{9} / \mathrm{L}$ (OR 2.7, $p=0.0007$ ), albumin below normal (OR 2.1, $\mathrm{p}=0.01$ ), LDH above normal (OR 3.9, $p<0.0001)$, an advanced directive of no CPR (OR 10.8, $p<0.0001)$ and having an ICU admission (OR 8.3, $p<0.0001$ ) (Table 3 and Fig. 1).

\section{Discussion}

ICU morbidity and mortality remain an important issue for patients with hematologic malignancy. Although predictors of mortality in patients admitted to ICU with hematologic malignancy are well described ${ }^{1,4-6,11-22}$, there is a paucity of evidence that identifies the predictors of ICU admission in this population, or predictors of survival in hospitalized patients with hematologic malignancies. Overall, during a period of 5.5 years, $19 \%$ of patients with hematologic malignancies admitted to our tertiary care hospital were admitted to an ICU at least once, with $47 \%$ of these admissions to a high acuity (versus step-down) ICU.

Interestingly, we found that the predictors of ICU admission in hospitalized patients with hematologic cancers were a combination of disease-related (acute leukemia, curative intent chemotherapy), laboratory-related (platelet count, albumin, LDH), and physician-related factors (having advanced directives discussion). The latter factor likely represents the fact that physicians are more likely to have goals of care discussions in patients with impending or actual deterioration. The disease-related factors of acute leukemia and curative intent chemotherapy likely represent the more aggressive philosophy of care that patients and physicians have in diseases with curative potential. 


\begin{tabular}{|c|c|c|c|c|}
\hline & Total $(\mathrm{N}=\mathbf{8 2 0})$ & Non-ICU admission $(n=641)$ & ICU admission $(n=179)$ & $p$ value \\
\hline Age at admission (years) & & & & 0.16 \\
\hline $\mathrm{N}$ & 820 & 641 & 179 & \\
\hline Median (inter-quartiles) & $66(56,76)$ & $66(56,76)$ & $64(55,74)$ & \\
\hline Min, $\max$ & 18,98 & 18,98 & 19,96 & \\
\hline BMI $\left(\mathrm{kg} / \mathrm{m}^{2}\right)$ & & & & 0.58 \\
\hline $\mathrm{N}$ & 709 & 577 & 132 & \\
\hline Median (inter-quartiles) & $25.7(22.1,30.3)$ & $25.7(22.1,30.6)$ & $25.8(22.0,29.9)$ & \\
\hline Min, $\max$ & $7.3,127.5$ & $7.3,127.5$ & $14.7,44.2$ & \\
\hline Gender & & & & 0.23 \\
\hline Male & $462(56.3 \%)$ & $354(55.2 \%)$ & $108(60.3 \%)$ & \\
\hline Female & $358(43.7 \%)$ & $287(44.8 \%)$ & $71(39.7 \%)$ & \\
\hline Time from diagnosis to admission (months) & & & & 0.0002 \\
\hline $\mathrm{N}$ & 819 & 640 & 179 & \\
\hline Median (inter-quartiles) & $6.7(2.3,36.9)$ & $7.0(2.8,41.3)$ & $5.6(0.8,27.0)$ & \\
\hline Min, $\max$ & $0.0,328.7$ & $0.0,328.7$ & $0.0,243.6$ & \\
\hline $\begin{array}{l}\text { Time from last chemotherapy to admission } \\
\text { (months) }\end{array}$ & & & & 0.62 \\
\hline $\mathrm{N}$ & 614 & 505 & 109 & \\
\hline Median (inter-quartiles) & $1(0,3)$ & $1(0,3)$ & $1(0,4)$ & \\
\hline Min, $\max$ & 0,232 & 0,232 & 0,113 & \\
\hline Reason for admission $(n=819)$ & & & & 0.02 \\
\hline Febrile neutropenia, infection, IP & $289(35.3 \%)$ & $219(34.2 \%)$ & $70(39.3 \%)$ & \\
\hline $\begin{array}{l}\text { Systemic (metabolic, CVS, HEME, RESP, GI, } \\
\text { GU, Neuro, orthopaedic) }\end{array}$ & $257(31.4 \%)$ & $201(31.4 \%)$ & $65(36.3 \%)$ & \\
\hline $\begin{array}{l}\text { Directly cancer related (e.g. Chemotherapy, } \\
\text { workup, ASCT, relapse) }\end{array}$ & $184(22.4 \%)$ & $152(23.7 \%)$ & $32(18.0 \%)$ & \\
\hline Pain & $33(4.0 \%)$ & $30(4.7 \%)$ & $3(1.7 \%)$ & \\
\hline FTT & $56(6.8 \%)$ & $48(7.5 \%)$ & $8(4.5 \%)$ & \\
\hline Any RBC transfusion preceding admission & & & & 0.013 \\
\hline No & $622(75.9 \%)$ & $499(77.9 \%)$ & $123(68.7 \%)$ & \\
\hline Yes & $198(24.2 \%)$ & $142(22.2 \%)$ & $56(31.3 \%)$ & \\
\hline PLT transfusion preceding admission & & & & 0.58 \\
\hline No & $733(89.4 \%)$ & $575(89.7 \%)$ & $158(88.3 \%)$ & \\
\hline Yes & $87(10.6 \%)$ & $66(10.3 \%)$ & $21(11.7 \%)$ & \\
\hline Chemotherapy intent & & & & $<.0001$ \\
\hline Curative & $648(79.0 \%)$ & $529(82.5 \%)$ & $119(66.5 \%)$ & \\
\hline Non-curative & $172(21.0 \%)$ & $112(17.5 \%)$ & $60(33.5 \%)$ & \\
\hline Chemotherapy line & & & & 0.26 \\
\hline $\mathrm{N}$ & 641 & 506 & 135 & \\
\hline Median (inter-quartiles) & $1(1,2)$ & $1(1,2)$ & $2(1,3)$ & \\
\hline Min, $\max$ & 1,7 & 1,6 & 1,7 & \\
\hline Chemotherapy pre-hospital admission & & & & 0.31 \\
\hline No & $179(21.8 \%)$ & $135(21.1 \%)$ & $44(24.6 \%)$ & \\
\hline Yes & $641(78.2 \%)$ & $506(78.9 \%)$ & $135(75.4 \%)$ & \\
\hline Acute leukemia & & & & $<.0001$ \\
\hline No & $709(86.5 \%)$ & $575(89.7 \%)$ & $134(74.9 \%)$ & \\
\hline Yes & $111(13.5 \%)$ & $66(10.3 \%)$ & $45(25.1 \%)$ & \\
\hline Histology details $(n=809)$ & & & & $<.0001$ \\
\hline Lymphoid-very aggressive & $52(6.4 \%)$ & $36(5.7 \%)$ & $16(8.9 \%)$ & \\
\hline Lymphoid-aggressive & $327(40.4 \%)$ & $265(42.1 \%)$ & $62(34.6 \%)$ & \\
\hline Lymphoid-indolent & $199(24.6 \%)$ & $166(26.4 \%)$ & $33(18.4 \%)$ & \\
\hline Myeloid-very aggressive & $87(10.8 \%)$ & $50(7.9 \%)$ & $37(20.7 \%)$ & \\
\hline Myeloid-aggressive & $60(7.4 \%)$ & $48(7.6 \%)$ & $12(6.7 \%)$ & \\
\hline Myeloid-indolent & $4(0.5 \%)$ & $4(0.6 \%)$ & $0(0 \%)$ & \\
\hline Plasma cell neoplasm & $80(9.9 \%)$ & $61(9.7 \%)$ & $19(10.6 \%)$ & \\
\hline \multicolumn{5}{|l|}{ Laboratory values } \\
\hline Hemoglobin & & & & $<.0001$ \\
\hline Continued & & & & \\
\hline
\end{tabular}




\begin{tabular}{|c|c|c|c|c|}
\hline & Total $(\mathrm{N}=820)$ & Non-ICU admission $(n=641)$ & ICU admission $(\mathrm{n}=179)$ & $p$ value \\
\hline $\mathrm{N}$ & 819 & 640 & 179 & \\
\hline Median (inter-quartiles) & $95(81,113)$ & $96(83,115)$ & $89(75,106)$ & \\
\hline Min, $\max$ & 26,161 & 26,161 & 45,152 & \\
\hline Platelet count & & & & $<.0001$ \\
\hline $\mathrm{N}$ & 817 & 640 & 177 & \\
\hline Median (inter-quartiles) & $121(43,216)$ & $135(50,224)$ & $74(22,159)$ & \\
\hline Min, $\max$ & 1,979 & 1,979 & 2,598 & \\
\hline White blood cells & & & & 0.46 \\
\hline $\mathrm{N}$ & 820 & 641 & 179 & \\
\hline Median (inter-quartiles) & $4.7(1.7,9.0)$ & $4.6(1.8,8.6)$ & $5.0(1.5,10.8)$ & \\
\hline Min, $\max$ & $0.0,666.0$ & $0.0,553.4$ & $0.0,666.0$ & \\
\hline Creatinine clearance & & & & 0.008 \\
\hline $\mathrm{N}$ & 702 & 572 & 130 & \\
\hline Median (inter-quartiles) & $78(55,111)$ & $80(57,111)$ & $69(42,104)$ & \\
\hline Min, $\max$ & 4,352 & $11.4,352.3$ & $3.9,226.2$ & \\
\hline Albumin & & & & $<.0001$ \\
\hline $\mathrm{N}$ & 761 & 591 & 170 & \\
\hline Median (inter-quartiles) & $36(32,40)$ & $37(33,40)$ & $33(29,39)$ & \\
\hline Min, $\max$ & 3,51 & 3,51 & 15,47 & \\
\hline LDH & & & & $<.0001$ \\
\hline $\mathrm{N}$ & 497 & 416 & 81 & \\
\hline Median (inter-quartiles) & $240(182,383)$ & $231(177,346)$ & $353(206,653)$ & \\
\hline Min, $\max$ & 0,8175 & 0,8175 & 0,4500 & \\
\hline \multicolumn{5}{|c|}{ Modified Charlson Comorbidity Index (CCI) } \\
\hline Modified CCI & & & & 0.02 \\
\hline $\mathrm{N}$ & 820 & 641 & 179 & \\
\hline Median (inter-quartiles) & $1(0,2)$ & $1(0,2)$ & $1(0,2)$ & \\
\hline Min, $\max$ & 0,12 & 0,12 & 0,10 & \\
\hline Congestive heart failure & & & & 0.10 \\
\hline No & $714(87.1 \%)$ & $565(88.1 \%)$ & $149(83.2 \%)$ & \\
\hline Yes & $106(12.9 \%)$ & $76(11.9 \%)$ & $30(16.8 \%)$ & \\
\hline PVD & & & & 0.01 \\
\hline No & $718(87.6 \%)$ & $571(89.1 \%)$ & $147(82.1 \%)$ & \\
\hline Yes & $102(12.4 \%)$ & $70(10.9 \%)$ & $32(17.9 \%)$ & \\
\hline Cerebrovascular disease & & & & 0.09 \\
\hline No & $774(94.4 \%)$ & $610(95.2 \%)$ & $164(91.6 \%)$ & \\
\hline Yes & $46(5.6 \%)$ & $31(4.8 \%)$ & $15(8.4 \%)$ & \\
\hline Dementia & & & & 0.09 \\
\hline No & $801(97.7 \%)$ & $623(97.2 \%)$ & $178(99.4 \%)$ & \\
\hline Yes & $19(2.3 \%)$ & $18(2.8 \%)$ & $1(0.6 \%)$ & \\
\hline Diabetes with end organ damage & & & & 0.61 \\
\hline No & $797(97.2 \%)$ & $624(97.4 \%)$ & $173(96.6 \%)$ & \\
\hline Yes & $23(2.8 \%)$ & $17(2.6 \%)$ & $6(3.4 \%)$ & \\
\hline Diabetes without end organ damage & & & & 0.91 \\
\hline No & $697(85.0 \%)$ & $544(84.9 \%)$ & $153(85.5 \%)$ & \\
\hline Yes & $123(15.0 \%)$ & $97(15.1 \%)$ & $26(14.5 \%)$ & \\
\hline Renal disease & & & & 0.012 \\
\hline No & $700(85.4 \%)$ & $558(87.0 \%)$ & $142(79.3 \%)$ & \\
\hline Yes & $120(14.6 \%)$ & $83(13.0 \%)$ & $37(20.7 \%)$ & \\
\hline Liver disease (mild) & & & & 0.74 \\
\hline No & $760(92.7 \%)$ & $595(92.8 \%)$ & $165(92.2 \%)$ & \\
\hline Yes & $60(7.3 \%)$ & $46(7.2 \%)$ & $14(7.8 \%)$ & \\
\hline Pulmonary disease & & & & 0.02 \\
\hline No & $764(93.2 \%)$ & $605(94.4 \%)$ & $159(88.8 \%)$ & \\
\hline Yes & $56(6.8 \%)$ & $36(5.6 \%)$ & $20(11.2 \%)$ & \\
\hline Solid cancer with metastases & & & & 0.40 \\
\hline Continued & & & & \\
\hline
\end{tabular}




\begin{tabular}{|l|l|l|l|l|}
\hline & Total $(\mathrm{N}=\mathbf{8 2 0})$ & Non-ICU admission $(\mathbf{n}=\mathbf{6 4 1})$ & ICU admission $(\mathbf{n}=\mathbf{1 7 9})$ & $\boldsymbol{p}$ value \\
\hline No & $786(95.9 \%)$ & $612(95.5 \%)$ & $174(97.2 \%)$ & \\
\hline Yes & $34(4.2 \%)$ & $29(4.5 \%)$ & $5(2.8 \%)$ & \\
\hline Goals of care discussion in hospital pre-ICU & & & & $<.0001$ \\
\hline No & $518(63.2 \%)$ & $433(67.6 \%)$ & $85(47.5 \%)$ & \\
\hline Yes & $302(36.8 \%)$ & $208(32.4 \%)$ & $94(52.5 \%)$ & \\
\hline Advance directive of not to attempt CPR & & & & 0.089 \\
\hline No & $704(85.9 \%)$ & $543(84.7 \%)$ & $161(89.9 \%)$ & \\
\hline Yes & $116(14.2 \%)$ & $98(15.3 \%)$ & $18(10.1 \%)$ & \\
\hline
\end{tabular}

Table 1. Demographic characteristics of patients. $B M I$ body mass index, $I P$ infection-pneumonia, $H E M E$ hematologic, RESP respiratory, GI gastrointestinal, GU genitourinary, ASCT autologous stem cell transplant, FTT failure to thrive, $C V S$ cardiovascular, $R B C$ red blood cell $P V D$ peripheral vascular disease, $I C U$ intensive care unit, $C P R$ cardiopulmonary resuscitation. Bonferroni adjusted $p$ value $<0.002$ was considered statistically significant (bold).

\begin{tabular}{|l|l|l|l|l|l|}
\hline Final model & $\boldsymbol{p}$ value & OR & $\mathbf{9 5 \%}$ CI of OR & & Model fit $\mathbf{R}^{\mathbf{2}}$ (\%) \\
\hline Intercept & $<.0001$ & & & & \\
\hline Chemotherapy intent (palliative vs. curative) & $\mathbf{0 . 0 2}$ & 2.09 & 1.13 & 3.78 \\
\hline Platelet count $<50 \times 10^{9} /$ L (yes vs. no) & $\mathbf{0 . 0 4}$ & 1.76 & 1.00 & 3.06 & \multirow{2}{*}{10.56} \\
\hline Acute leukemia (yes vs. no) & $\mathbf{0 . 0 0 0 4}$ & 3.32 & 1.70 & 6.39 \\
\hline Albumin below normal (yes vs. no) & $\mathbf{0 . 0 1}$ & 1.97 & 1.16 & 3.35 \\
\hline LDH above normal (yes vs. no) & $\mathbf{0 . 0 2}$ & 1.85 & 1.09 & 3.20 \\
\hline Advanced directive discussion in hospital pre ICU (yes vs. no) & $\mathbf{0 . 0 1}$ & 1.95 & 1.15 & 3.32 & \\
\hline
\end{tabular}

Table 2. Multivariable logistic regression model of predictors of ICU admission.

It is noteworthy that ICU admission was independently associated with an eight times greater risk of death within 30 days compared to other admitted patients. Forty percent of ICU-admitted patients died in hospital, with patients presenting with an increased $\mathrm{LDH}$ and those requiring mechanical ventilation at greatest risk for death. The early and short-term mortality rates for ICU admitted patients were high (cumulative mortality rates at 1 month 34\% and at 6 months 53\%). This is similar to previous data, which report a range of in-hospital mortality of 46-90\% $\%^{1,4-6,11-22}$, with multi-organ dysfunction (illustrated by use of mechanical ventilation, vasopressor use, renal replacement therapy, significant liver enzyme elevation, high Acute Physiologic Assessment and Chronic Health Evaluation (APACHE) II, Sequential Organ Failure Assessment (SOFA), or Simplified Acute Physiology Score (SAPS) scores) being the most common predictor of death ${ }^{1,4-6,11-22}$. This was further confirmed in larger studies including a literature review of observational studies encompassing 10,000 patients ${ }^{23}$, and a retrospective analysis of 7689 patients with hematologic malignancy admitted to the ICU ${ }^{24}$. In fact, this is similar to the contemporary literature of predictive factors for mortality in general medical patients admitted to $\mathrm{ICU}^{25}$. However, this is the first time that LDH has been reported as an independent risk factor for mortality in patients with hematologic malignancy.

Notably, we did not find that body mass index, infection as a reason for admission, disease histology, presence of leukemia, recent chemotherapy, chemotherapy intent, line of chemotherapy, cytopenias, albumin, or comorbidity index score to be associated with an increased risk of death, which had been reported by others ${ }^{17,23}$. This may reflect the smaller sample size of ICU admitted patients in this analysis or the selection bias inherent with offering patients with acute leukemia induction chemotherapy. Of interest is one study that reported that the prognosis of a patient's hematologic malignancy does not affect ICU mortality ${ }^{26}$, while others found that leukemia, recent transplant, Hodgkin lymphoma, or untreated malignancy were independent risk factors for mortality ${ }^{3,27}$; furthermore, hematologic malignancy is one variable of the SAPS score.

The cumulative mortality rate for non-ICU admitted patients was not low ( 1 month $12 \%, 6$ months $28 \%$ ) suggesting that any admission for toxicity or diagnosis related to a hematologic cancer is associated with inferior survival. Other factors independently predictive of death in hospital or within 1 month of discharge for all patients were: age, PLT count $<50 \times 10^{9} / \mathrm{L}$, reduced albumin, elevated $\mathrm{LDH}$, and an advanced directive of no CPR. Apart from the advanced directives, these clinical and laboratory variables are valuable for counselling patients, families and health care providers especially since only $53 \%$ of ICU admitted and $33 \%$ of non-ICU admitted patients had advanced directives discussions documented in the charts.

There are several limitations to our study. This was a retrospective study, which may be limited by selection bias and incomplete data although sequential admissions were captured accurately by health data specialists. It is possible that advanced directive discussions occurred that were not documented in the electronic or paper patient chart, although this seems unlikely, and both were reviewed by chart audit. We did not record the 

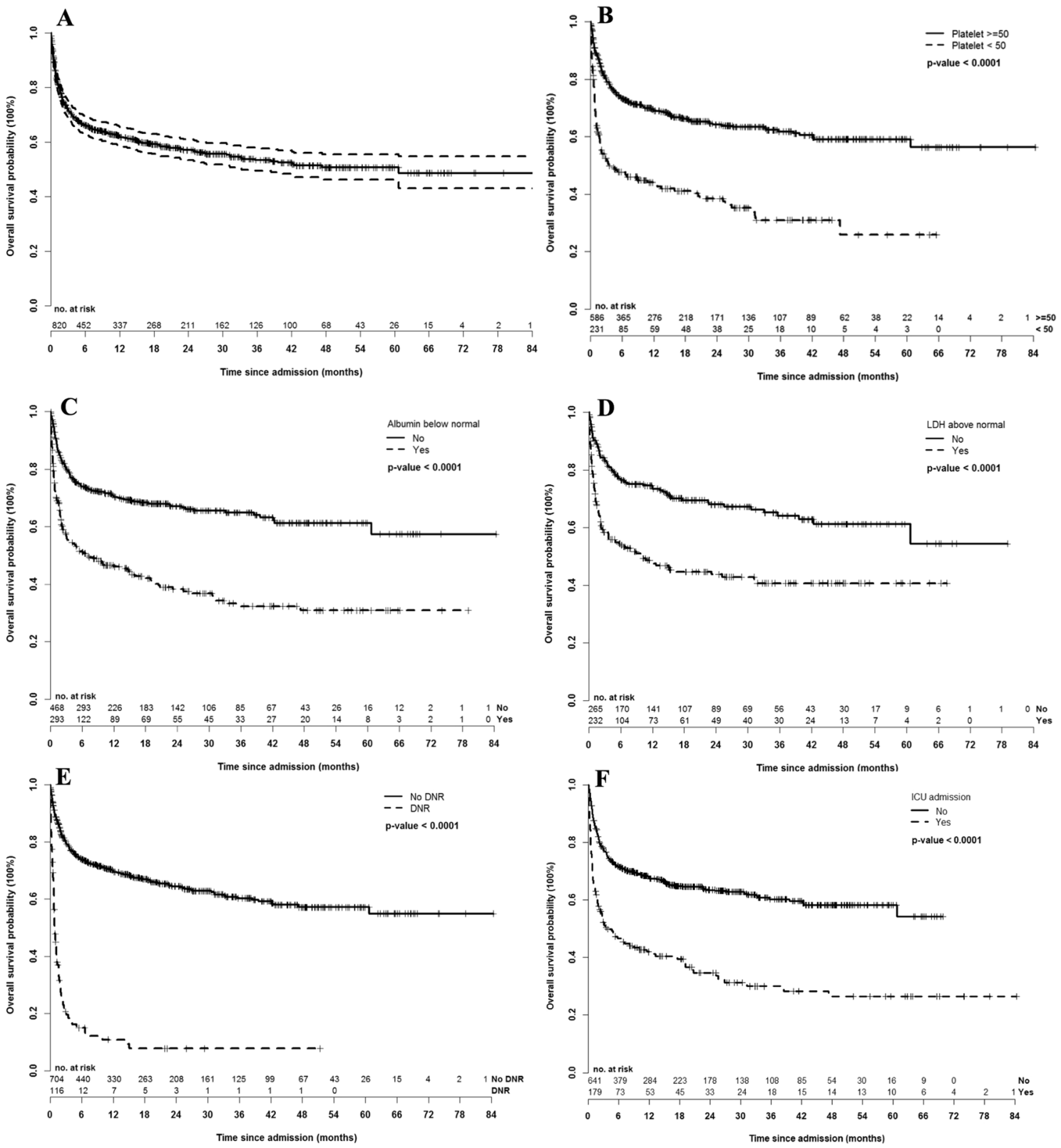

Figure 1. (A) Overall survival of all patients; (B) overall survival stratified by platelet count $<50 \times 10^{9} / \mathrm{L}$ or $\geq 50 \times 10^{9} / \mathrm{L} ;(\mathbf{C})$ overall survival stratified by albumin above or below normal; (D) overall survival stratified by lactate dehydrogenase (LDH) above or below normal; (E) overall survival stratified by do not resuscitate (DNR) status; (F) overall survival stratified by intensive care unit admission (yes/no).

\begin{tabular}{|c|c|c|c|c|c|}
\hline Final model & $p$ value & OR & $95 \% \mathrm{CI}$ of OR & & Model fit $\mathbf{R}^{2}(\%)$ \\
\hline Intercept & $<.0001$ & & & & \multirow{7}{*}{31.25} \\
\hline Age at admission (years) & 0.004 & 1.03 & 1.01 & 1.05 & \\
\hline Platelet count at admission $<50 \times 10^{9} / \mathrm{L}$ (yes vs. no) & 0.0007 & 2.70 & 1.52 & 4.82 & \\
\hline Albumin below normal at admission (yes vs. no) & 0.01 & 2.09 & 1.18 & 3.70 & \\
\hline LDH above normal at admission (yes vs. no) & $<.0001$ & 3.93 & 2.19 & 7.28 & \\
\hline $\begin{array}{l}\text { No cardiopulmonary resuscitation order documented at anytime during } \\
\text { hospitalization (yes vs. no) }\end{array}$ & $<.0001$ & 10.84 & 5.46 & 22.16 & \\
\hline ICU admission (yes vs. no) & $<.0001$ & 8.26 & 4.30 & 16.21 & \\
\hline
\end{tabular}

Table 3. Multivariable logistic regression model of predictors of death during hospitalization or within 1 month of discharge in all hospitalized patients. 
timing of the advanced directives discussion and many may have occurred very near to clinical deterioration and potential death.

To be consistent among all patients, the laboratory values we considered were those upon admission to hospital and not during the hospital stay, which can increase or decrease with time and may be more frequently assayed in the sicker patients admitted to the ICU. Since admission to ICU from hospitalization was short, we believe there may still be some value in considering these admission laboratory parameters. We did not have APACHE, SOFA or SAPS scores to consider, which undoubtedly contributed to the paucity of predictors for mortality related to ICU admitted patients.

The strengths of our study include a large sample size of 870 patients with a heterogeneous collection of hematologic cancers and our unique focus on the predictors of ICU admission and overall survival in all patients admitted to hospital with hematologic cancers, which to our knowledge has never been reported before. As more patients with hematological malignancy are surviving and undergoing treatment, it becomes increasingly important to identify not only which patients will be admitted to ICU, but which patients may benefit from an intensive care unit admission.

The best discussions with patients about risk of mortality and morbidity occur outside of crisis situations. The predictive factors for ICU admission that we determined at our institution to be important at the time of hospitalization (non-curative intent chemotherapy, PLT $<50 \times 10^{9} / \mathrm{L}$, acute leukemia, low albumin, elevated LDH) may remind clinicians about the need for goals of care discussions before deterioration occurs. With the advent of big data, artificial intelligence and machine learning algorithms, one could envision a predictive score being generated in hospitalized patients, which could be used to guide clinical practice including the more careful monitoring of higher risk patients and complete documentation of goals of care.

Received: 20 January 2020; Accepted: 19 November 2020

Published online: 03 December 2020

\section{References}

1. Cornish, M., Butler, M. B. \& Green, R. S. Predictors of poor outcomes in critically ill adults with hematologic malignancy. Can. Respir. J. 2016, 9431385. https://doi.org/10.1155/2016/9431385 (2016).

2. Grgic Medic, M., Gornik, I. \& Gasparovic, V. Hematologic malignancies in the medical intensive care unit-outcomes and prognostic factors. Hematology 20, 247-253. https://doi.org/10.1179/1607845414Y.0000000206 (2015).

3. Yeo, C. D. et al. Prognostic factors in critically ill patients with hematologic malignancies admitted to the intensive care unit. J. Crit. Care 27(739), e731-736. https://doi.org/10.1016/j.jcrc.2012.07.014 (2012).

4. Benoit, D. D., Vandewoude, K. H., Decruyenaere, J. M., Hoste, E. A. \& Colardyn, F. A. Outcome and early prognostic indicators in patients with a hematologic malignancy admitted to the intensive care unit for a life-threatening complication. Crit. Care Med. 31, 104-112. https://doi.org/10.1097/01.CCM.0000038213.27741.30 (2003).

5. Geerse, D. A., Span, L. F., Pinto-Sietsma, S. J. \& van Mook, W. N. Prognosis of patients with haematological malignancies admitted to the intensive care unit: Sequential Organ Failure Assessment (SOFA) trend is a powerful predictor of mortality. Eur. J. Intern. Med. 22, 57-61. https://doi.org/10.1016/j.ejim.2010.11.003 (2011).

6. Bird, G. T., Farquhar-Smith, P., Wigmore, T., Potter, M. \& Gruber, P. C. Outcomes and prognostic factors in patients with haematological malignancy admitted to a specialist cancer intensive care unit: a 5 yr study. Br. J. Anaesth. 108, 452-459. https://doi. org/10.1093/bja/aer449 (2012).

7. Hill, Q. A. et al. Survival of patients with hematological malignancy admitted to the intensive care unit: prognostic factors and outcome compared to unselected medical intensive care unit admissions, a parallel group study. Leuk. Lymphoma 53, 282-288. https://doi.org/10.3109/10428194.2011.614705 (2012).

8. Hawari, F. I., Nazer, L. H., Addassi, A., Rimawi, D. \& Jamal, K. Predictors of ICU admission in patients with cancer and the related characteristics and outcomes: a 5-year registry-based study. Crit. Care Med. 44, 548-553. https://doi.org/10.1097/CCM.00000 00000001429 (2016).

9. Gruson, D. et al. Predictive factors of intensive care unit admission in patients with haematological malignancies and pneumonia. Intensive Care Med. 30, 965-971. https://doi.org/10.1007/s00134-004-2237-1 (2004).

10. Deyo, R. A., Cherkin, D. C. \& Ciol, M. A. Adapting a clinical comorbidity index for use with ICD-9-CM administrative databases. J. Clin. Epidemiol. 45, 613-619 (1992).

11. Sawicka, W., Owczuk, R., Wujtewicz, M. A. \& Wujtewicz, M. The effectiveness of the APACHE II, SAPS II and SOFA prognostic scoring systems in patients with haematological malignancies in the intensive care unit. Anaesthesiol. Intensive Ther. 46, 166-170. https://doi.org/10.5603/AIT.2014.0030 (2014).

12. Parakh, S. et al. Outcomes of haematology/oncology patients admitted to intensive care unit at The Canberra Hospital. Intern. Med. J. 44, 1087-1094. https://doi.org/10.1111/imj.12545 (2014).

13. Namendys-Silva, S. A., Gonzalez-Herrera, M. O., Texcocano-Becerra, J. \& Herrera-Gomez, A. Outcomes of critically ill gynecological cancer patients admitted to intensive care unit. Am. J. Hosp. Palliat. Care 30, 7-11. https://doi.org/10.1177/104990911243702 8 (2013).

14. Lamia, B. et al. Changes in severity and organ failure scores as prognostic factors in onco-hematological malignancy patients admitted to the ICU. Intensive Care Med. 32, 1560-1568. https://doi.org/10.1007/s00134-006-0286-3 (2006).

15. Kroschinsky, F. et al. Outcome and prognostic features of intensive care unit treatment in patients with hematological malignancies. Intensive Care Med. 28, 1294-1300. https://doi.org/10.1007/s00134-002-1420-5 (2002).

16. Horster, S. et al. Mortality of patients with hematological malignancy after admission to the intensive care unit. Onkologie 35, 556-561. https://doi.org/10.1159/000342672 (2012).

17. Cherif, H., Martling, C. R., Hansen, J., Kalin, M. \& Bjorkholm, M. Predictors of short and long-term outcome in patients with hematological disorders admitted to the intensive care unit for a life-threatening complication. Support. Care Cancer 15, 1393-1398. https://doi.org/10.1007/s00520-007-0268-1 (2007).

18. Ferra, C. et al. Outcome and prognostic factors in patients with hematologic malignancies admitted to the intensive care unit: a single-center experience. Int. J. Hematol. 85, 195-202. https://doi.org/10.1532/IJH97.E0625 (2007).

19. Faucher, E. et al. Short- and long-term outcomes in onco-hematological patients admitted to the intensive care unit with classic factors of poor prognosis. Oncotarget 7, 22427-22438. https://doi.org/10.18632/oncotarget.7986 (2016).

20. Evison, J. et al. Intensive care unit admission in patients with haematological disease: incidence, outcome and prognostic factors. Swiss Med. Wkly. 131, 681-686 (2001). 
21. Cornet, A. D. et al. Sequential organ failure predicts mortality of patients with a haematological malignancy needing intensive care. Eur. J. Haematol. 74, 511-516. https://doi.org/10.1111/j.1600-0609.2005.00418.x (2005).

22. Azoulay, E. et al. Outcomes of critically ill patients with hematologic malignancies: prospective multicenter data from France and Belgium-a groupe de recherche respiratoire en reanimation onco-hematologique study. J. Clin. Oncol. 31, 2810-2818. https:// doi.org/10.1200/JCO.2012.47.2365 (2013).

23. McDowall, K. L., Hart, A. J. \& Cadamy, A. J. The outcomes of adult patients with haematological malignancy requiring admission to the intensive care unit. Intensive Care Soc. 12, 112-125 (2011).

24. Hampshire, P. A., Welch, C. A., McCrossan, L. A., Francis, K. \& Harrison, D. A. Admission factors associated with hospital mortality in patients with haematological malignancy admitted to UK adult, general critical care units: a secondary analysis of the ICNARC Case Mix Programme Database. Crit. Care 13, R137. https://doi.org/10.1186/cc8016 (2009).

25. Fika, S., Nanas, S., Baltopoulos, G., Charitidou, E. \& Myrianthefs, P. A novel mortality prediction model for the current population in an adult intensive care unit. Heart Lung 47, 10-15. https://doi.org/10.1016/j.hrtlng.2017.10.009 (2018).

26. Massion, P. B. et al. Prognosis of hematologic malignancies does not predict intensive care unit mortality. Crit. Care Med. 30, 2260-2270. https://doi.org/10.1097/01.CCM.0000030456.11264.EF (2002).

27. Silfvast, T., Pettila, V., Ihalainen, A. \& Elonen, E. Multiple organ failure and outcome of critically ill patients with haematological malignancy. Acta Anaesthesiol. Scand. 47, 301-306 (2003).

\section{Acknowledgements}

The authors wish to acknowledge Liying Zhang for her statistical analysis.

\section{Author contributions}

N.C., D.J., Z.F., M.C., S.G., R.F., and R.B. conceptualized the project. N.C., D.J., Z.F., R.B. curated the data. A.V., R.B. interpreted the data and wrote the original draft. All authors reviewed and edited the final manuscript.

\section{Funding}

There were no funding sources for this project.

\section{Competing interests}

The authors declare no competing interests.

\section{Additional information}

Supplementary information is available for this paper at https://doi.org/10.1038/s41598-020-78114-7.

Correspondence and requests for materials should be addressed to R.B.

Reprints and permissions information is available at www.nature.com/reprints.

Publisher's note Springer Nature remains neutral with regard to jurisdictional claims in published maps and institutional affiliations.

(c) (i) Open Access This article is licensed under a Creative Commons Attribution 4.0 International

License, which permits use, sharing, adaptation, distribution and reproduction in any medium or format, as long as you give appropriate credit to the original author(s) and the source, provide a link to the Creative Commons licence, and indicate if changes were made. The images or other third party material in this article are included in the article's Creative Commons licence, unless indicated otherwise in a credit line to the material. If material is not included in the article's Creative Commons licence and your intended use is not permitted by statutory regulation or exceeds the permitted use, you will need to obtain permission directly from the copyright holder. To view a copy of this licence, visit http://creativecommons.org/licenses/by/4.0/.

(C) The Author(s) 2020 